\title{
3D/TSV Enabling Technologies for SOC/NOC: Modeling and Design Challenges
}

\author{
Khaled Salah \\ Mentor Graphics \\ Cairo, Egypt. \\ Khaled_mohamed@mentor.com
}

\author{
Alaa El Rouby \\ Mentor Graphics \\ Cairo, Egypt.
}

Alaa_El-Rouby@mentor.com

\author{
Hani Ragai \\ Ain-Shams University \\ Cairo, Egypt. \\ Yehea Ismail \\ Nile University \\ Cairo, Egypt.
}

\begin{abstract}
According to the International Technology Roadmap for Semiconductors (ITRS), the traditional scaling will no longer meet the performance and integration requirements of systemson-chip (SoC) in the long term. Therefore, new I/O and packaging paradigms are needed. Three-dimensional integration is a promising alternative option to traditional 2D planar chips. 3D integration is mainly restricted by the communication infrastructure between different stacked dies of future multi-core SoC and network-on-chip (NoC). Among several 3D integration technologies, the TSV (Through-Silicon-Via) approach is the most promising one and therefore is the focus of the majority of 3D integration $R \& D$ activities. However, there are challenges that should be overcome before the production of TSV-based $3 \mathrm{D}$ ICs becomes possible, e.g., electrical modeling challenges, thermal and power challenges, technological challenges, design methodology challenges and CAD tool development challenges.
\end{abstract}

Index Terms - Three-Dimensional ICs, Through Silicon Via, Modeling, TSV, Macro-Modeling, Challenges.

\section{INTRODUCTION AND BACKGROUND}

$I^{\prime}$ ncreasing drive for the integration of different signals (analog, digital, RF) and technologies (SOI, HBTs, GaAs, strained silicon, and so on) is introducing various design concepts, for which existing planar (2D) technologies may not be suitable, so, three-dimensional (3D) integrated circuits is introduced. A qualitative comparison regarding the benefits introduced by the $3 \mathrm{D}$ integration process, compared to existing system design approaches is summarized in Table I. 3D integration technology provides increased performance in many design criteria as compared to the current 2D approaches, as 3D-ICs, which contain multiple layers of active devices, extensively utilize the vertical dimension to connect components and are expected to address interconnect delay related problems (In planar (2D) technologies, scaling is reducing gate delays but also increasing interconnect ones and this will be mitigated by the use of short wires in 3D designs, as schematically shown in Fig.1. These shorter wires will decrease the average load capacitance and resistance and decrease the number of repeaters which are needed to regenerate signal on long wires) and to enable integration of heterogeneous technologies (in the 3D design, a 2D chip is divided into a number of different blocks, and each one is placed on a separate layer of silicon where each layer is stacked on top of each other, as schematically shown in Fig.2. This may be exploited to build SoC by placing different circuits with performance requirements in different layers which enables novel "More than Moore "applications) [1,2].

In 3-D integration, the communication among the stacked ICs and embedded components requires vertical interconnections. Since processing the vertical interconnections is challenging compared to the planar ones, the electrical and mechanical characteristics of the vertical interconnections can be a bottleneck for achieving the required system performance. Thus, various types of vertical interconnections are still being proposed. Several interconnection elements are already popular in industry. Fig.3 shows typical 3D structures with four types of interconnections, including bonding wires, TSV interconnections (which is a new silicon-based 3-D packaging structure), metal bumps and contactless communication. Table II summarizes the main difference between those four types of interconnects. It is clear that to improve electrical performance. TSV interconnections are becoming a major choice to replace the bonding wires and metal bumps [3]. TSV is not new, but now it is finally an option [4].

TABLE I

COMPARISON BETWEEN ALTERNATIVE DESIGN IMPLEMENTATIONS

\begin{tabular}{|c|c|c|c|}
\hline Property & Single chips & SOC & 3D \\
\hline Modular flexibility & High & Low & Medium \\
\hline System performance & Low & Medium & High \\
\hline Physical dimension & Large & Medium & Small \\
\hline Complexity of fabrication & Low & Medium & Medium \\
\hline Cost & Low & Medium & High \\
\hline $\begin{array}{c}\text { CAD tools and design } \\
\text { methodologies }\end{array}$ & Available & Available & $\begin{array}{c}\text { Not deployed } \\
\text { yet }\end{array}$ \\
\hline
\end{tabular}

TABLE II

COMPARISON AMONG DIFFERENT 3D ASSEMBLY TECHNOLOGIES

\begin{tabular}{|c|c|c|}
\hline Technologies & Advantages & Disadvantages \\
\hline Wire bonding & $\begin{array}{c}\text {-Flexible connection } \\
\text {-high reliability } \\
\text {-their processing is } \\
\text { mature and cost } \\
\text { effective }\end{array}$ & $\begin{array}{c}\text {-Low density } \\
\text {-Long wiring } \\
\text {-Poor signal integrity } \\
\text {-Large pad area }\end{array}$ \\
\hline metal bumps & -Short length & -Large solder ball \\
\hline TSVs & $\begin{array}{c}\text {-Short length } \\
\text {-High density } \\
\text {-Small footprint }\end{array}$ & -Complex Fabrication \\
\hline Contactless & $\begin{array}{c}\text { reducing the length } \\
\text { of electrical paths } \\
\text { (inductive and capacitive } \\
\text { coupling) }\end{array}$ & $\begin{array}{c}\text {-less reliability } \\
\text {-cross talk and } \\
\text { coupling issues } \\
\text {-size of inductors } \\
\text { (inductive coupling) }\end{array}$ \\
\hline
\end{tabular}


$\sqrt{\mathrm{A}}$

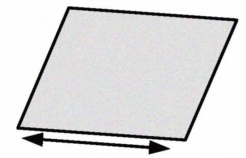

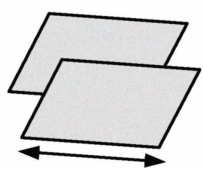

$\sqrt{\mathrm{A} / 2}$

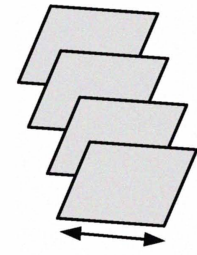

$\sqrt{\mathrm{A} / 4}$

Area $=\mathrm{A}$
$\mathrm{L}=\sqrt{\mathrm{A} / 4}$
Area $=\mathrm{A}$$$
\text { Area }=\mathrm{A}
$$

$L=\sqrt{A / 2}$

Fig. 1 Reduction in wire length where the original 2-D circuit is implemented in two and four planes.

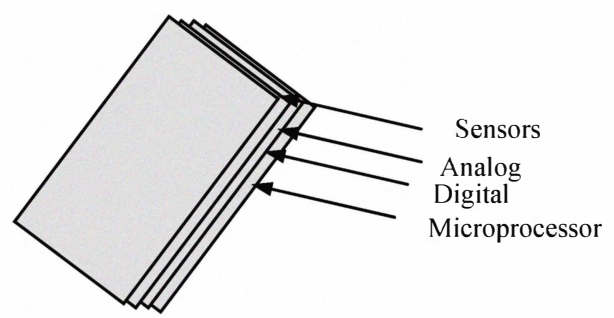

Fig. 2 A possible design for a 3D SoC (heterogeneous technologies).

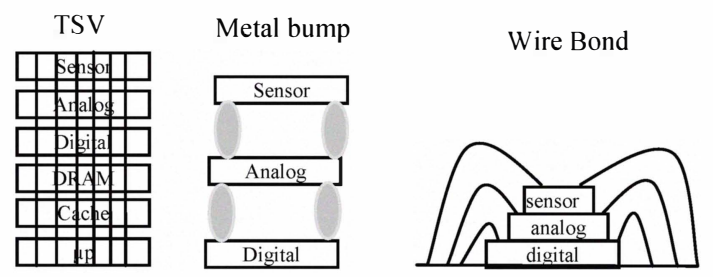

Contactless (capacitive and inductive coupling)

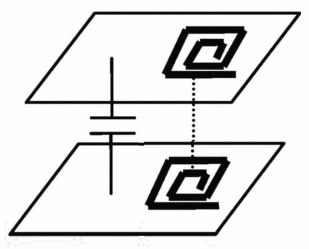

Fig. 3 Examples of 3D structures with interconnection elements

The general structure of a TSV (Fig. 4) is typically a cylinder with a uniform circular cross-section of a conducting material (copper) surrounded by an insulator (silicon dioxide) which is intended to prevent leakage and resistive coupling through the substrate (silicon). 3D integrated circuits, which contain multiple layers of active devices, extensively utilize the vertical dimension to connect components. Usually, the TSV interconnections are fabricated as an array in a silicon substrate. TSVs distribution is mainly determined during physical design such as partitioning, placement, and routing. TSVs can be laid out in a row, within a regular matrix, or within an irregular distribution depending on the applications and technology constraints [1]-[4]. The characteristics of a TSV are dependent on its geometrical parameters (such as the TSV diameter, height, pitch, and oxide layer thickness) and

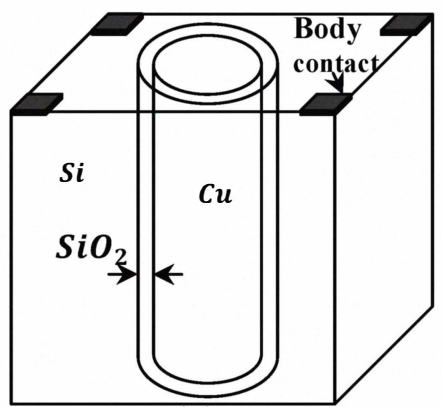

(a)

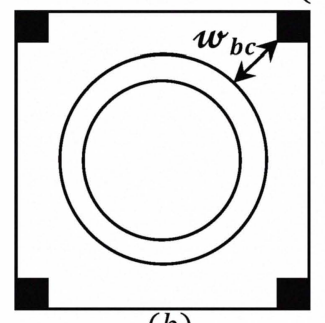

(b)

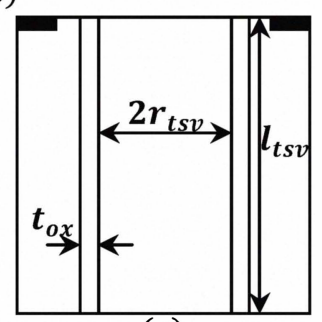

(c)
Fig. 4 The through silicon via structure assuming a uniform circular cross section of cupper surrounded by an oxide inside a silicon substrate (a) 3D view (b) Top view (c) Side view.

electrical parameters (such as the metal conductivity, oxide permittivity, and the silicon substrate resistivity).

3D integration technology using TSVs is a relatively new research field. In this paper, we addresses technology trends for 3D integration, highlight the 3D/TSV modeling and design challenges, and review existing solutions. The rest of this paper is organized as follows. In Section II, the challenges of TSV-based 3D integration are discussed. Conclusions are given in Section III.

\section{MODELING AND DESIGN CHALLENGES FOR 3D ICS}

Although 3D ICs offer many advantages over 2D ICs, many challenges should be overcome before TSV-based 3D ICs production becomes possible. These challenges levels are shown in Fig. 5, and are discussed below:

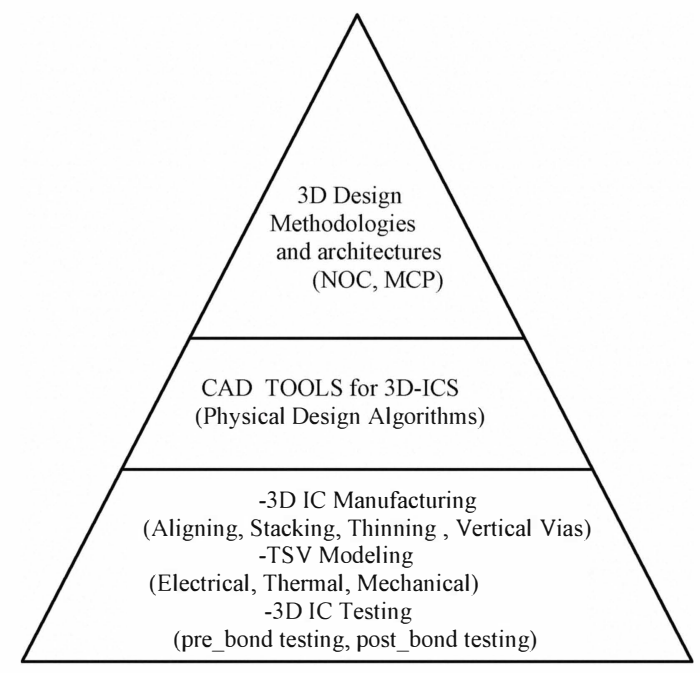

Fig. 5 3D IC: the complete solution and challenges. 


\section{A. Electrical Modeling Challenges}

The purpose of modeling interconnections is to extract equivalent circuits that describe the electrical characteristics of given interconnection structures. A major difficulty in modeling 3-D interconnections comes from the need to obtain the entire coupling model of a large number of 3D interconnections. Furthermore, for accurate electrical design of 3D ICs including radio frequency (RF), analog, and digital submodules, the 3-D interconnection model should cover a sufficiently wide frequency range. The broadband model needs to capture frequency-dependent losses, coupling, and mismatch, which are contributed by the parasitic elements such as series inductances, resistances, shunt capacitances, and conductance. Unlike conventional interconnects, a TSV is surrounded by the silicon substrate that has a finite resistivity. This finite resistivity results in a nonlinear capacitance and resistance parasitics that are not present in conventional interconnect, and a large capacitance that is highly dependent on TSV relative position to nearby body contact. Therefore, in case of modeling TSV interconnections, the silicon substrate should be considered as another source of loss. Clearly, TSV modeling is extremely challenging when a large number of TSV interconnections are involved. A typical lumped model of a single interconnection is composed of a series inductor, a series resistor, and a parallel capacitor. The values of circuit elements are determined by the shape of the interconnection itself as well as the coupling from nearby interconnections and other passive structures such as power/ground planes. The TSV is a more general structure than transmission lines . Thus, applying transmission line theory for 3-D interconnection modeling is not appropriate. Because of this fundamental difficulty with 3-D interconnection modeling, most previous research relied on indirect ways for characterization based on measurements, analytical methods, and numerical methods. The measurement-based equivalent model is difficult to extend to general multi-TSV structures due to the increased number of model parameters that need to be tuned to the multi-port measurement data. So the numerical methods are used[3].

One of the problems being researched in this domain is the modeling of multi-TSV arrangement in a 3-D system and their time-domain co-simulation in a simulator like SPICE. Most of previous work characterizes specific TSV structures but they do not address general multi-TSV problems [5]-[15]. Other related work proposes a model but no closed-form expressions are given for the TSV parasitic calculations [19]. In other words, there has been no related work in TSV macromodeling. Also, few work addresses the multi-stacked TSV modeling problem.

\section{B. Thermal Management Challenges}

Thermal management is one of the important issues of 3D IC integration. So, effective thermal management methodologies and solutions are needed for widespread use of 3D IC integration. The thermal analysis is an important as from the thermal and stress distribution, we can make layout modification and thus enhance the circuit reliability. The thermal issue of a 3D IC is much severer than that of a $2 \mathrm{D} \mathrm{IC.}$ The cause is that the ambient environment of the die of a $2 \mathrm{D}$ IC is the cooling material, but the ambient environment of a die within a 3D IC may be another die that also generates heat. Therefore, Thermal management in 3D ICs is critical for maintaining required reliability, performance, and power dissipation target. Therefore, new cooling techniques must be innovated to ensure that the chip can operate at temperatures and performance required for reliability $[20,21]$.

\section{CAD Tools Challenges}

The addition of a third dimension would require support from more advanced CAD tools (floorplan gets $3^{\text {rd }} \mathrm{D}$ ). 3D IC physical design has attracted an increasing amount of attention. There is a significant amount of work on the floorplanning, placement and routing for 3D ICs. However, all these tools developed by different groups, using different formats to represent the design data, create barriers for researchers who need to make use of the existing design automation tools to conduct further studies on 3D IC. Also, there are a lack of CAD algorithms and only few commercially available EDA tools for 3D integrated circuits. Therefore, current 2D physical design tools (partitioning, placement, routing, timing, extraction, LVS, DRC) must enable 3D designs[22,23].

\section{Technological, Yield and Test Challenges}

There is new types of defects caused by TSV formation, and alignment (The alignment accuracy or bonding quality (including wafer to-wafer alignment)), a broad range of open issues are in a pressing need for novel ideas. Solutions are needed to address these challenges before 3D integration technology can be widely used [24]. Also, It is well known that yield has a quadratic dependency on die size, and a linear dependency on chip count at a given die size. 3D designs may incur some yield loss due to vertical vias, and may gain some yield due to density [25]. There is still a lack of pre-bond test, post-bond test and DFT solutions.

\section{E. Design Methodologies and Circuit Architectures Challenges}

3D design methodologies are still needed to reduce coupling between TSVs (body contacts reduces the current loop so it reduces the mutual inductances) and for clock and power distribution networks for 3D ICs. Also, more investigations are required for analyzing the effect of TSVs on signal integrity, power integrity and delay. The 3-D integration also provides opportunities for new circuit architectures. For example, a 2-D FPGA requires 2-D interconnections that grow faster than gate count. Thus, 2-D FPGAs are limited in performance by the complexities of their internal interconnections. A 3-D FPGA would overcome this 
interconnect limitations, resulting in faster data throughput, and faster switching of the gate-level configuration.

Table III summarizes the $3 \mathrm{D}$ challenges and their related work status [26, 27].

TABLE III

3DCHALLENGES AND THEIR RELATED WORK STATUS

\begin{tabular}{|c|l|}
\hline Challenge & \multicolumn{1}{|c|}{ Related Work Status } \\
\hline Electrical Modeling & Research initiated, some models are available \\
\hline Thermal Modeling & $\begin{array}{l}\text { Research initiated, some tools and techniques are } \\
\text { available. }\end{array}$ \\
\hline 3D CAD Tools & $\begin{array}{l}\text { No complete solution is available, research } \\
\text { needed }\end{array}$ \\
\hline Yield and Test & No solution is available, research is needed \\
\hline Technological & Nearly Mature \\
\hline $\begin{array}{c}\text { Design } \\
\text { Methodologies }\end{array}$ & $\begin{array}{l}\text { No complete solution is available, research is } \\
\text { needed }\end{array}$ \\
\hline
\end{tabular}

\section{CONCLUSIONS}

3D integration technology using TSV is one of possible future IC design technologies. It can offer many advantages over the existing 2D integration technology. However, many challenges (modeling, thermal, CAD tools, yield and test, technological, design methodologies, circuit architectures) should be overcome before TSV-based 3D ICs production becomes possible.

\section{REFERENCES}

[1] K.Siozios, A.Bartzas,D.Soudris" Architecture-Level Exploration of Alternative Interconnection Schemes Targeting 3D FPGAs: A Software-Supported Methodology" International Journal of Reconfigurable Computing, Volume 2008

[2] Dae Hyun Kim, Saibal Mukhopadhyay, and Sung Kyu Lim, "TSVaware Interconnect Length and Power Prediction for 3D Stacked ICs", IEEE International Interconnect Technology Conference, pp. 2628, 2009.

[3] K. Banerjee, S. J. Souri, P. Kapur, and K. C. Saraswat, "3-d ics: A novel chip design for improving deep-submicrometer interconnect performance and systems-on-chip integration," Proc. IEEE, vol. 89, no. 5, pp. 602-633, May 2001 .

[4] K.P.Stuby,"Hourglass-shaped conductive connection through semiconductor structures," US. Pat. No. 3648131 Assignee: IBM, Filing Date: Nov. 7, 1969, Issue Date: Mar. 7, 1972

[5] C. Ryu, D. Chung, Junho Lee, K. Lee, T. Oh, J. Kim, "High frequency electrical circuit model of chip-to-chip vertical via interconnection for 3-D chip stacking package," in IEEE 14th Topical Meeting on Electrical Performance of Electronic Packaging, Oct. 2005, pp. 151154.

[6] J. Pak, C. Ryu, J. Kim, "Electrical Characterization of Through Silicon Via (TSV) depending on Structural and Material Parameters based on 3D Full Wave Simulation," in Electromagnetic Electronic Materials and Packaging, 2007.Conf., May.2008, pp. 351-354.

[7] C. Ryu, J. Lee, H. Lee, K. Lee, T. Oh, J. Kim, "High Frequency Electrical Model of Through Wafer Via for 3- D Stacked Chip Packaging," in Electronics System integration Technology Conf, vol. 1, Sept. 2006, pp. 215-220.

[8] Dong Min Jang, Chunghyun Ryu, Kwang Yong Lee, Byeong Hoon Cho, Joungho Kim, Tae Sung Oh, Won Jong Lee, and Jin Yu, "Development and Evaluation of 3- D SiP with Vertically Interconnected Through Silicon Vias (TSV)," in Proc. of ECTC, 2007, pp. 847-852.

[9] Chunghyun Ryu, Joungho Kim Jun So Pak, "Multi-Stacked ThroughSilicon-Via Effects on Signal Integrity and Power In tegrity for
Application of 3-Dimensional Stacked-Chip-Package,".

[10] Soon Wee Ho, Seung Wook Yoon, Qiaoer Zhou, Krishnamachar Pasad, Vaidyanathan Kripesh and John H. Lau, "High RF Performance TSV Silicon Carrier for High Frequency Application," in Electronic Components and Technology Conference, 2008.

[11] Adam Beece, Kenneth Rose, Tong Zhang, and Jian Qiang Lu Zheng $\mathrm{Xu}$, "Modeling and Evaluation for Electrical Characteristics of Through-Strata-Vias (TSVs) in Three-Dimensional Integration," in 3D System Integration, 2009. 3DIC 2009. IEEE International Conference.

[12] Jongjoo Shim, Eakhwan Song, Jun So Pak, Junho Lee, Hyungdong Lee, Kunwoo Park, and Joungho Kim Jonghyun Cho, "Active Circuit to Through Silicon Via (TSV) Noise Coupling," Electrical Performance of Electronic Packaging and Systems, 2009. EPEPS '09. IEEE 18th Conference

[13] J.Kim, E.Song, J.Cho, J.Pak, J. Lee, H. Lee, K.Park, and J. Kim, "Through Silicon Via (TSV) Equalizer," in Electrical Performance of Electronic Packaging and Systems, 2009. EPEPS '09. IEEE 18th Conference.

[14] M. Rousseau, O. Rozeau, G. Cibrario, G. Le Carval, M.-A. Jaud, P Leduc, A. Farcy, A. Marty, "Through-silicon via based 3D IC technology: Electrostatic simulations for design methodology," in IMAPS Device Packaging Conference, Phoenix, AZ : United States, 2008

[15] I. Savidis and E. G. Friedman, "Electrical Modeling and Characterization of 3-D Vias," in Proc. IEEE Int. Symp. Circuits Syst., May 2008.

[16] Ioannis Savidis and E. G. Friedman, "Closed-Form Expressions of 3-D Via Resistance, Inductance, and Capacitance," IEEE TRANSACTIONS ON ELECTRON DEVICES, vol. 56, no. 9, SEPTEMBER 2009.

[17] R. Weerasekera, D. Pamunuwa, M. Grange, H. Tenhunen, and L.-R. Zheng, "Closed-form Equations for Through-Silicon Via (TSV) Parasitics in 3-D Integrated Circuits (ICs)," in Proc. Workshop 3-D Integr., DATE Conf., Apr. 2009

[18] E.Eid, T. Lacrevaz, S. de Rivaz, C. Bermond, B. Flchet, F. Calmon, C. Gontrand, A. Farcy, L.Cadix, and P. Ancey, "Predictive High Frequency Effects of Substrate Coupling in 3D Integrated Circuits Stacking," in 3D System Integration, 2009. 3DIC 2009.

[19] R. Weerasekera, D. Pamunuwa, M. Grange, H. Tenhunen, and L.-R Zheng, "Closed-Form Equations for Through-Silicon Via(TSV) Parasitics in 3-D Integrated Circuits," in Proc. Workshop 3-D Integr., DATEConf., Apr. 2009.

[20] P.Leduca, F.d.Crécya, M.Fayollea, B.Charleta, T.Enota, M.Zussya, B Jonesb, J.C.Barbea, N.Kerneveza,S. Maitrejeana, D.Louisa, G.Passemardc "Challenges for 3D IC integration: bonding quality and thermal managementc". 2007 IEEE

[21] T.-Y. Chiang, S. J. Souri, C. O. Choi, and K. C. Saraswat, "Thermal analysis of heterogeneous 3-D ICs with various integration schemes," in Proc. IEEE Electron Devices Meeting, 2001, pp. 681-684.

[22] Chiang, C. and Sinha, S.: "The Road to 3D EDA Tool Readiness, Proc" 2009 Conference on Asia and South Pacific Design Automation, pp.429-436 (2009).

[23] A. Rahman and R. Reif. "System-level performance evaluation of three-dimensional integrated circuits." IEEE Trans. on VLSI Systems, Special Issue on System-Level Interconnect Prediction, 8(6):671-678, 2000.

[24] K. Bernstein, P. Andry, J. Cann, Ph. Emma, D. Greenberg, W Haensch, M. Ignatowski, S. Koester, J. Magerlein, R Puri, A. Young, " Interconnects in the Third Dimension: Design Challenges for 3D ICs," Proc. 44th Annual Conference on Design Automation, pp.562-567 (2007).

[25] C. Ferri, S. Reda and I. Bahar, "Parametric yield management for 3D ICs: Models and strategies for improvement," ACM Journal on Emerging Technologies in Computing Systems, vol. 4, 2008.

[26] Jin-Fu Li, Cheng-Wen Wu, " Is 3D Integration an Opportunity or Just a Hype?," in IEEE 2009.

[27] Luca P. Carloni, Partha Pande, Yuan Xie , " Networks-on-Chip in Emerging Interconnect Paradigms: Advantages and Challenges," in IEEE 2009. 